Revue des patrimoines

43 | 2021

Des écoles d'art académiques aux écoles d'art : des collections et des lieux, un patrimoine à valoriser

\title{
Exposer la collection anatomique de l'École des beaux-arts de Dresde
}

To exhibit the anatomical collection of the Dresden University of Fine Arts: the German federal project "Körper und Malerei"

BMBF-Projekt „Körper und Malerei“": Erschließung, Erforschung und Nutzung der Anatomischen Lehrsammlung und der Gemäldesammlung der Hochschule für Bildende Künste Dresden

\section{Sandra Mühlenberend, Jakob Fuchs et Ivo Mohrmann}

Traducteur : Isabelle Chave

\section{OpenEdition} Journals

Édition électronique

URL : http://journals.openedition.org/insitu/30207

DOI : 10.4000/insitu.30207

ISSN : 1630-7305

Éditeur

Ministère de la Culture

Référence électronique

Sandra Mühlenberend, Jakob Fuchs et Ivo Mohrmann, « Exposer la collection anatomique de l'École des beaux-arts de Dresde », In Situ [En ligne], 43 | 2021, mis en ligne le 02 février 2021, consulté le 03 février 2021. URL : http://journals.openedition.org/insitu/30207 ; DOI : https://doi.org/10.4000/insitu. 30207

Ce document a été généré automatiquement le 3 février 2021.

In Situ Revues des patrimoines est mis à disposition selon les termes de la licence Creative Commons Attribution - Pas d'Utilisation Commerciale - Pas de Modification 4.0 International. 


\title{
Exposer la collection anatomique de l'École des beaux-arts de Dresde
}

\author{
To exhibit the anatomical collection of the Dresden University of Fine Arts: the \\ German federal project "Körper und Malerei" \\ BMBF-Projekt „Körper und Malerei“: Erschließung, Erforschung und Nutzung \\ der Anatomischen Lehrsammlung und der Gemäldesammlung der Hochschule \\ für Bildende Künste Dresden
}

Sandra Mühlenberend, Jakob Fuchs et Ivo Mohrmann

Traduction : Isabelle Chave

\section{L'École supérieure des beaux-arts de Dresde}

1 L'École supérieure des beaux-arts de Dresde est l'une des plus anciennes académies des Beaux-Arts d'Europe. Une école de dessin et de peinture fut créée de façon pionnière dès 1680. En 1764, l'École supérieure a été fondée sous le nom de Haupt-KunstAkademie. Peu de temps après sa fondation, cette Académie des beaux-arts réussit à attirer des enseignants de renommée nationale et internationale pour l'enseignement de ses étudiants. Parmi les plus connus figurent, entre autres, Charles-François Hutin, Bernardo Bellotto, Giuseppe Camerata, Giovanni Battista Casanova et Lorenzo Zucchi ainsi qu'Anton Graff et Adrian Zingg.

$2 \mathrm{Au}$ XIX ${ }^{\mathrm{e}}$ siècle, l'académie attira à Dresde de nombreux artistes de renom tels que Caspar David Friedrich, Philipp Otto Runge et Georg Friedrich Kersting. Mentionnons en particulier la longue et importante activité de Ludwig Richter, qui étudia à l'académie puis y enseigna la peinture à partir de 1836. L'architecte Gottfried Semper, qui joua un rôle décisif dans la création du paysage urbain de Dresde dans la première moitié du XIX ${ }^{e}$ siècle, devint professeur au département d'Architecture en 1834.

3 La seconde moitié du XIXe siècle fut une période de stagnation. Afin d'échapper à la rigidité de la formation académique, l'Académie des beaux-arts nomma l'impressionniste Gotthardt Kuehl professeur de peinture en 1895. D'importants 
professeurs tels Otto Gussmann, Carl Bantzer, Eugen Bracht, Robert Sterl, Ferdinand Dorsch, et des étudiants comme Max Pechstein, Kurt Schwitters, George Grosz, Conrad Felixmüller et Otto Dix suivirent. En quelques années, l'Académie des beaux-arts se renouvela complètement et, dans les années 1920, son importance fut renforcée par la nomination d'Oskar Kokoschka et Otto Dix ${ }^{1}$.

4 Après la Seconde Guerre mondiale, en 1947, l'académie reprit ses activités d'enseignement. En 1950, elle fusionna avec l'École des arts et métiers pour former la Hochschule für Bildende Künste (École supérieure des beaux-arts). L'objectif fut alors de faire de cette école un centre de formation artistique du réalisme socialiste. Jusqu'en 1990, plusieurs réformes furent engagées, toujours en rapport avec les orientations et les besoins de la société. L'art figuratif continua à être au centre de l'attention et ce n'est que vers la fin des années 1980 qu'il fut possible de travailler sur l'art abstrait ${ }^{2}$.

\section{Le projet de recherche fédéral « Körper und Malerei »}

Sous l'intitulé «Interconnecter, développer, chercher », le ministère fédéral allemand de l'Éducation et de la Recherche (BMBF) a lancé en 2016 un projet coopératif pour les collections universitaires. Grâce au financement de ce programme, deux collections uniques de l'École supérieure des beaux-arts (Hochschule für Bildende Künste Dresden, HfBK) de Dresde sont sorties de l'ombre : la collection d'anatomie, qui compte environ 700 modèles, spécimens et planches pédagogiques et a été constituée de façon continue depuis 1800 , et la collection de quelque 1400 peintures, issues de mémoires de diplômes d'art et de projets de recherche d'étudiants réalisés entre 1950 et 1990.

Le projet «Körper und Malerei » («Corps et Peinture ») comprenait, entre autres, la réalisation d'un inventaire, d'un état des lieux et des mesures de conservationrestauration et avait pour ambition l'étude, la sauvegarde et la diffusion de ces deux collections, dont l'importance historique et la pertinence actuelle avaient été négligées jusqu'alors. En outre, le projet avait pour objectif de rendre les collections accessibles et exploitables pour la recherche et l'enseignement au-delà de la durée du projet (trois ans) en développant une infrastructure moderne. Si, pour la collection d'anatomie, on dispose bien d'une vue d'ensemble de la majorité des objets précieux et de leur usage à travers le temps ${ }^{3}$, le potentiel de la collection de peintures pour la recherche et l'enseignement avait été jusque-là pratiquement inexploité. L'importance des dépôts de diplômes d'art à l'École supérieure des beaux-arts et des travaux d'étudiants n'avait jusqu'à présent été reconnue ni par l'histoire de l'art, pour la peinture en République démocratique allemande, ni par les recherches sur l'histoire des institutions. Le travail sur et avec la collection de peintures avait également pour objectif de la revivifier par la recherche et l'enseignement.

7 Les problématiques actuelles dans le domaine de l'histoire du corps et du traitement éthique des spécimens humains historiques, des stratégies éducatives des académies d'art, de la matérialité, de l'histoire et de la signification des objets dans le contexte de collections scientifiques et artistiques et leurs liens complexes ont été constamment au centre de l'attention. Ce projet de trois ans a été accompagné et soutenu par son partenaire, les Staatliche Kunstsammlungen de Dresde (SKD, Collections nationales d'art de Dresde). L'étude pratique et théorique des deux collections s'est faite dans le cadre d'un dialogue interdisciplinaire entre l'histoire de l'art et l'histoire des sciences, la conservation-restauration, l'analyse scientifique et la gestion des collections. 


\section{La collection d'anatomie}

La collection d'anatomie de l'École supérieure des beaux-arts de Dresde est l'une des plus précieuses et des plus vastes en son domaine. Tous les éléments essentiels de l'anatomie artistique des deux derniers siècles y sont présents, qu'il s'agisse d'anatomie humaine ou animale [fig. 1, fig. 2]. Les champs d'étude qu'offrent environ 700 objets et plus de 1000 items individuels répondent aux recherches artistiques et scientifiques actuelles. La préservation de la collection repose sur plusieurs facteurs : l'enseignement continu de l'anatomie artistique de 1800 à nos jours, sa place insigne à l'École supérieure des beaux-arts de Dresde jusqu'aux années 1980 et un personnel professionnel et impliqué d'experts en médecine et dans les arts.

Figure 1

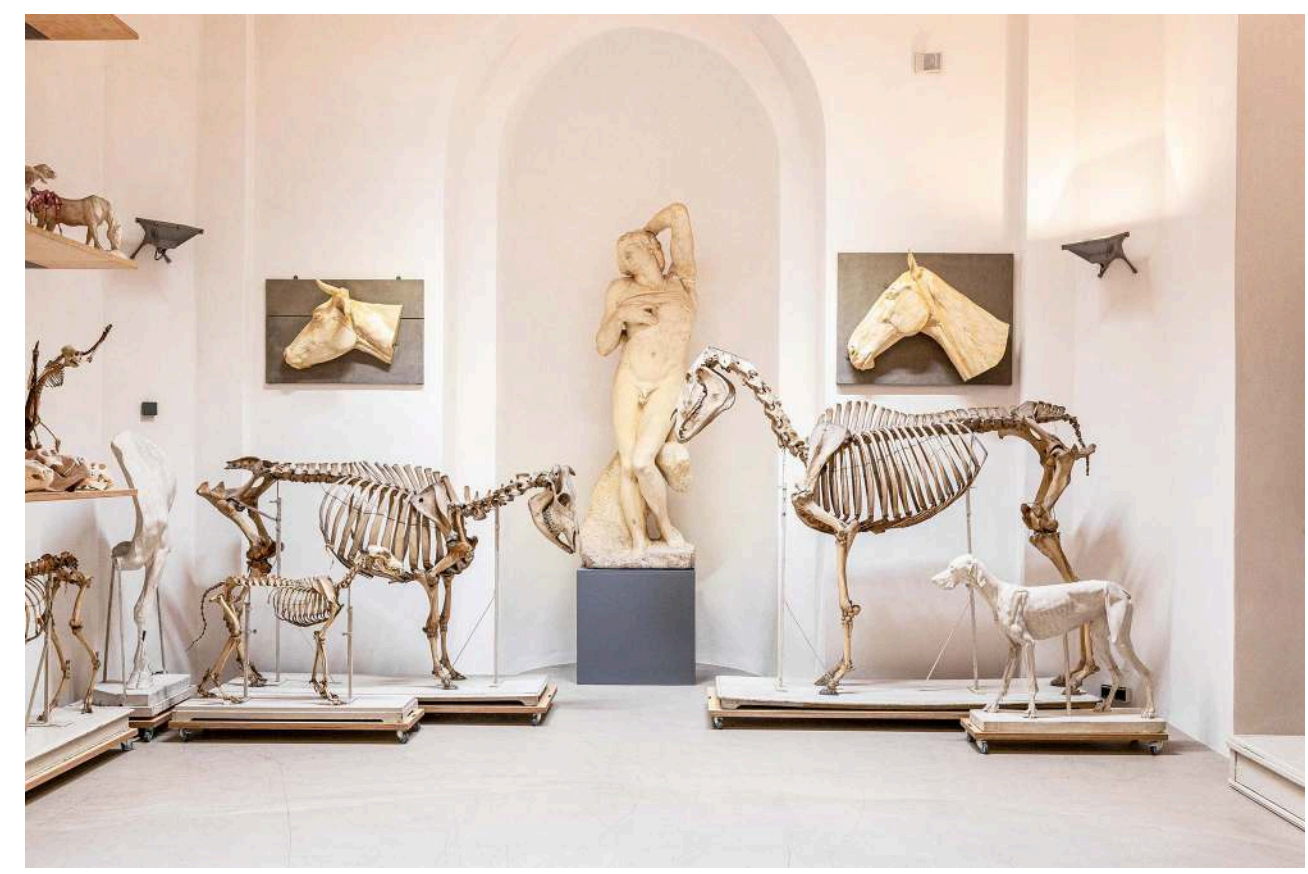

Modèles et spécimens d'anatomie animale et moulage en plâtre de L'Esclave mourant de Michel Ange. (c) Robert Vanis.

Dans l'histoire européenne de l'anatomie artistique, les disciplines de l'art et de la médecine furent étroitement liées dès l'origine. Tantôt deux représentants de l'une et de l'autre ont travaillé ensemble, tantôt les enjeux et les compétences de l'un ont embrassé le sujet proposé par l'autre. Ce lien particulier peut également s'observer à l'Académie des beaux-arts de Dresde dans le domaine de l'anatomie artistique. Celle-ci a d'abord été enseignée par des artistes, tels Giovanni Battista Casanova (1730-1795). Ce dernier, par intérêt artistique, s'est soucié de la structure interne du corps et a formulé un concept d'enseignement sur cette base. Il a été suivi plus tard par le célèbre anatomiste et médecin Burkhard Wilhelm Seiler (1779-1843) qui s'est non seulement tourné vers les arts mais a aussi conçu un manuel qui articulait ses connaissances et le goût artistique de son temps. Casanova et Seiler ont tous deux consulté des représentants de la discipline complémentaire, se sont laissé conseiller et ont ainsi pu développer leur propre stratégie de médiation. 


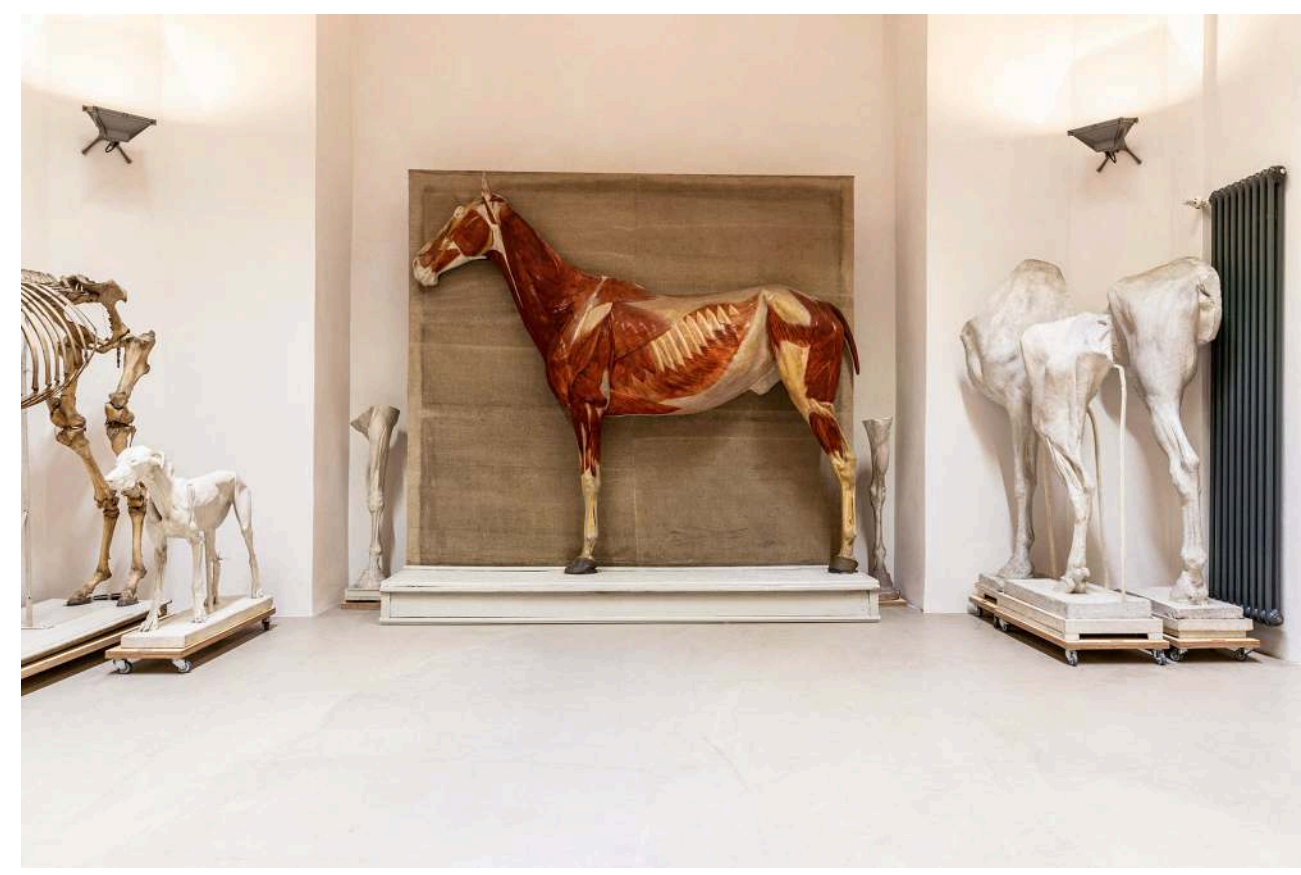

Relief de cheval polychromé grandeur nature en plâtre, probablement réalisé en collaboration avec l'école vétérinaire de Dresde entre 1900 et 1920.

(c) Robert Vanis.

La dotation en personnel de cette matière montre depuis sa création que ces professeurs avaient une carrière particulièrement longue, qu'ils étudiaient également les deux disciplines et qu'ils recherchaient et visualisaient des points d'ancrage avec le monde contemporain.

Parmi ces acteurs, Burkhard Wilhelm Seiler, Hermann Dittrich (1868-1946), Gottfried Bammes (1920-2007) et Manfred Zoller $\left({ }^{*} 1947\right)$ ont offert à l'anatomie artistique à l'Académie des beaux-arts de Dresde, et plus tard à l'École supérieure des beaux-arts, un succès durable. On leur doit, d'une part, l'accroissement de la collection, à travers des acquisitions et leurs propres conceptions d'objets anatomiques pédagogiques et d'autre part, l'élaboration de nouveaux concepts pédagogiques qui ont rayonné bien au-delà de l'académie et ont reçu une large reconnaissance ${ }^{4}$.

Une seule période chronologique paraît chaotique et faiblement dotée en personnel : la seconde moitié $\mathrm{du} \mathrm{XIX}^{\mathrm{e}}$ siècle, période où les réformes étaient plus que nécessaires C'est-à-dire une époque dans laquelle les réformes se faisaient plus qu'attendre et où l'Académie des beaux-arts dans son ensemble s'est figée. Sous le régime nazi, l'anatomie artistique s'est écartée du lien fécond entre les deux disciplines et l'accent était plutôt mis sur la représentation de proportions physiques surdimensionnées pour des raisons de propagande.

Le chapitre le plus sombre pour la collection anatomique correspond aussi aux années 1933 à 1945, qui sont de fait liées aux atrocités du national-socialisme. De 1933 à 1942, l'artiste Fritz Otto Sauerstein (1893-1968) a enseigné cette matière. Il a adapté ses leçons aux objectifs de l'idéologie nazie, introduisant notamment un cours sur les études raciales et la protection des races. Comme Dittrich, il disséquait des cadavres préalablement transférés à Dresde par la police des Affaires sociales du conseil 
municipal de Dresde. Il s'agissait de personnes décédées dont aucun parent ne s'était manifesté .

Cependant, Sauerstein a aussi demandé des dépouilles au centre d'exécution de la Münchner Platz. Il est prouvé qu'il a reçu un cadavre en 1936 et probablement aussi en 1941. Ce qu'il est advenu de ces cadavres après la dissection n'a pu être établi. Des enquêtes sur ces événements ont été menées en 2014 et 2017 et leurs résultats ont été consignés dans des rapports de recherche. Elles ont conduit à un réexamen des spécimens conservés dans la collection et à la décision du Sénat d'inhumer tous les restes humains isolés et qui ne pouvaient être affectés et de ne montrer que les squelettes reconstitués de la collection dont l'origine est extérieure à ce contexte inique ${ }^{6}$.

15 Parmi les spécimens non concernés par ce contexte d'injustice figurent ainsi sept squelettes à ligaments, dans une attitude de sculpture antique, conçus dans la première moitié du XIX $x^{e}$ siècle et uniques au monde ${ }^{7}$. Ils proviennent de l'ancienne collection de l'Académie royale de médecine et de chirurgie et ont été transférés à l'académie d'art après la dissolution de la collection en 1864. Les préparations très élaborées et les présentations particulières sont probablement attribuables à Burkhard Wilhelm Seiler. Les représentations de squelettes marquent l'apogée de la réflexion de l'Académie des beaux-arts sur l'idéal antique de beauté et les proportions propres aux sculptures antiques. Pour l'étude de l'antique, les squelettes à ligaments étaient placés dans l'attitude d'exempla préférés: par exemple, le Gladiateur Borghèse pour le corps fortement animé, le groupe du Laocoon (le squelette du Laocoon est perdu, les deux squelettes d'enfants ont été conservés) pour l'émotion et la passion, la Vénus Médicis pour la beauté de la figure féminine et le Tireur d'épine pour le calme et la concentration. Ces transpositions réussies servent ici à imiter et reproduire les modèles antiques vénérés alors comme l'anticipation d'un idéal esthétique ${ }^{8}$.

Outre ces importants supports et modèles d'anatomie humaine - tels des empreintes musculaires grandeur nature en plâtre et de rares crânes anatomiques en cire -, la collection contient aussi l'un des plus importants ensembles d'anatomie artistique animale. Certains de ces objets ont été achetés à des entreprises produisant du matériel pédagogique [fig. 3] ou légués par des institutions de Dresde9. Par ailleurs, les enseignants ont réalisé eux-mêmes des modèles originaux et des séries de spécimens en plâtre, parfois thématiques, et documentés par des photographies, des dessins et des publications. 
Figure 3

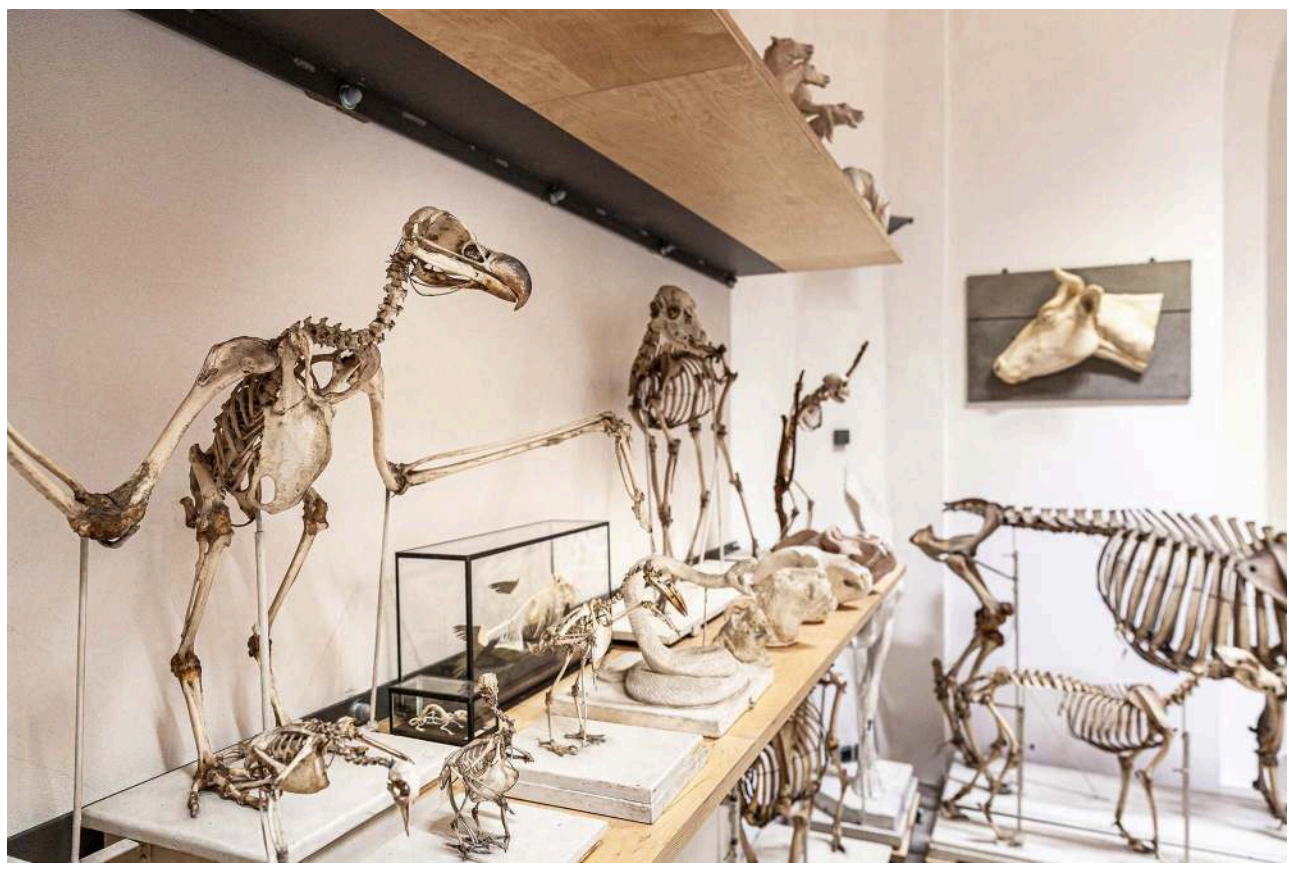

Modèles et spécimens d'anatomie animale : au premier plan, différents squelettes d'oiseaux.

(c) Robert Vanis.

17 Le torse musculaire de plâtre polychromé, grandeur nature, est un moulage original de la célèbre sculpture en bronze de Carl Schütz (dates de vie inconnues) conservée à l'Institut d'anatomie de la Charité à Berlin. C'est là qu'eut lieu le modelage préalable de la sculpture, en utilisant des préparations originales (cadavres) de l'Institut. L'anatomiste Hans Virchow (1852-1940), entre autres, participa à la réalisation de la sculpture, en documentant sa production en 1896. Son exécution ne se déroula pas sans difficultés et dura douze ans, avec la coopération d'autres artistes et anatomistes. Contrairement à la sculpture de bronze, les moulages en plâtre étaient directement destinés à servir de supports pédagogiques pour les établissements d'enseignement. La partie gauche du torse montre les muscles directement sous la couche de peau, la partie droite montre des structures plus profondes - et l'inverse, sur le crâne et les cuisses ${ }^{10}$.

La copie du moulage d'un tigre naturalisé a été réalisée vers 1900 à Munich dans l'atelier du sculpteur Willy Zügel (1876-1950). À côté de ses sculptures artistiques, Zügel a réalisé de nombreux moulages anatomiques en plâtre d'animaux, dont il a offert des tirages à divers établissements d'enseignement. Le tigre musclé est remarquable et constitue le premier modèle du genre; des académies des beaux-arts, principalement, en firent l'acquisition pour leurs collections anatomiques et comme modèle pour les représentations d'animaux.

En l'état actuel des connaissances, le moulage en plâtre de Dresde est le seul des exemplaires vendus (par exemple, aux académies des Beaux-Arts de Berlin et de Munich) qui ait été conservé ; le moulage d'après nature lui-même a aussi été perdu. Le tigre musclé est impressionnant, non seulement par son caractère de rareté, mais aussi par son exécution - position de marche, gueule ouverte, muscles tendus-, qui transforme la carcasse originelle en un prédateur vivant et agressif ${ }^{11}$. 

Ses propriétés matérielles, qui permettent de reproduire des éléments organiques en trompe-l'œil, ont fasciné les artistes et les anatomistes. Jusqu'au XIX ${ }^{e}$ siècle, elle était préférée pour la réalisation des modèles anatomiques. La collection contient, au total, 48 modèles anatomiques en cire: onze modèles en cire de membres supérieurs et inférieurs d'origine inconnue, vingt-deux reliefs anatomiques en cire d'après Josef Benedikt Kuriger (1754-1819) et quinze crânes anatomiques provenant de l'atelier de Carl Friedrich H. Heinemann (1802-1846). Les reliefs en cire et les crânes anatomiques ont aussi été déposés à l'Académie des beaux-arts après la dispersion de la collection de l'Académie royale de médecine et de chirurgie, en 1864.

21 Les modèles de bras et de jambes sont des supports d'étude classiques en anatomie artistique. Leur exécution très précise laisse penser qu'ils ont été reproduits en plâtre en grand nombre pour l'enseignement et ont été d'usage constant. On ne peut qu'émettre des hypothèses sur la manière dont les reliefs de cire et les crânes anatomiques étaient utilisés durant les cours d'anatomie artistique. Les reliefs de cire réalisés vers 1800 illustrent, certes, les connexions anatomiques mais plutôt à la façon des pièces d'un cabinet d'objets d'art. Les crânes anatomiques exécutés vers 1830 pour les médecins sont complètement différents; ils rendent les structures anatomiques les plus fines dans des parties précises de la tête en grandeur nature ou en taille supérieure ${ }^{12}$.

Outre cet ensemble de spécimens et de modèles, pour certains uniques, l'intérêt national et international de la collection d'anatomie est aussi dû au fait que des collections historiques comparables ont été perdues pour d'autres académies artistiques. Les seules exceptions sont la collection de l'École nationale supérieure des beaux-arts de Paris et celle de l'Académie russe des beaux-arts de Saint-Pétersbourg.

collection d'anatomie de Dresde, qui a débuté à la fin des années 1990 dans le cadre de la thèse de Sandra Mühlenberend ${ }^{13}$ et a conduit à une première sauvegarde de la collection, s'est accompagnée, comme à Paris et à SaintPétersbourg, du désir de lui assurer, avec ses objets variés et d'une grande fidélité de détails, une visibilité qu'elle n'a pas encore tout à fait actuellement et de la rendre de nouveau utilisable pour l'enseignement et la recherche. Avant que la collection d'anatomie puisse être à nouveau utilisée comme instrument scientifique et collection didactique à la HfBK de Dresde et présentée au public sous la forme d'expositions, elle a dû quitter un état de stockage désordonné, en partie non sécurisé, pour prendre une forme plus adéquate.

Il a donc fallu passer en revue et inventorier l'ensemble de la collection, documenter l'état des objets par leur analyse matérielle et, sur cette base, prendre des mesures de conservation et de restauration. Dans un premier temps, toute la collection a dû être retirée des deux salles principales, reliées entre elles. Comme les dépôts importants de poussière et de saleté étaient principalement dus à la maçonnerie de briques non crépie, toute la surface des murs des salles a été recouverte d'enduit. La fixation des étagères murales a été modifiée et un éclairage a été installé dans les espaces de travail et d'exposition.

Afin d'enregistrer les 468 spécimens et modèles de la collection à des fins de conservation et d'évaluer leur état, il a d'abord fallu élaborer un formulaire de protocole. Ce formulaire devait être utilisable pour dresser l'état de toute la collection et, outre la collecte des données de base et des détériorations, intégrer une brève 
analyse des détériorations (leur cause) et une analyse des risques (détériorations prévisibles à l'avenir). La très grande majorité des spécimens et des modèles a été examinée par inspection visuelle. Pour les précieuses études anatomiques de bras et de jambes, il a fallu réaliser des radiographies ${ }^{14}$ pour déterminer l'état et la composition des matériaux ${ }^{15}$ identifiés par l'analyse. Pour l'évaluation globale finale, six types de critères ont été définis ("état stable, sans détérioration", "état stable, avec détériorations partielles", "état stable, avec détériorations importantes", "état instable, avec détériorations partielles ", "état instable, avec détériorations importantes ", " état instable, sécurisation urgente »). Le critère de détérioration ainsi attribué a été saisi dans la base de données des archives. Les protocoles et les illustrations recto verso ${ }^{16}$, avec l'échelle de chaque objet, y sont également disponibles sous forme de fichiers aux formats PDF ou JPG.

Figure 4

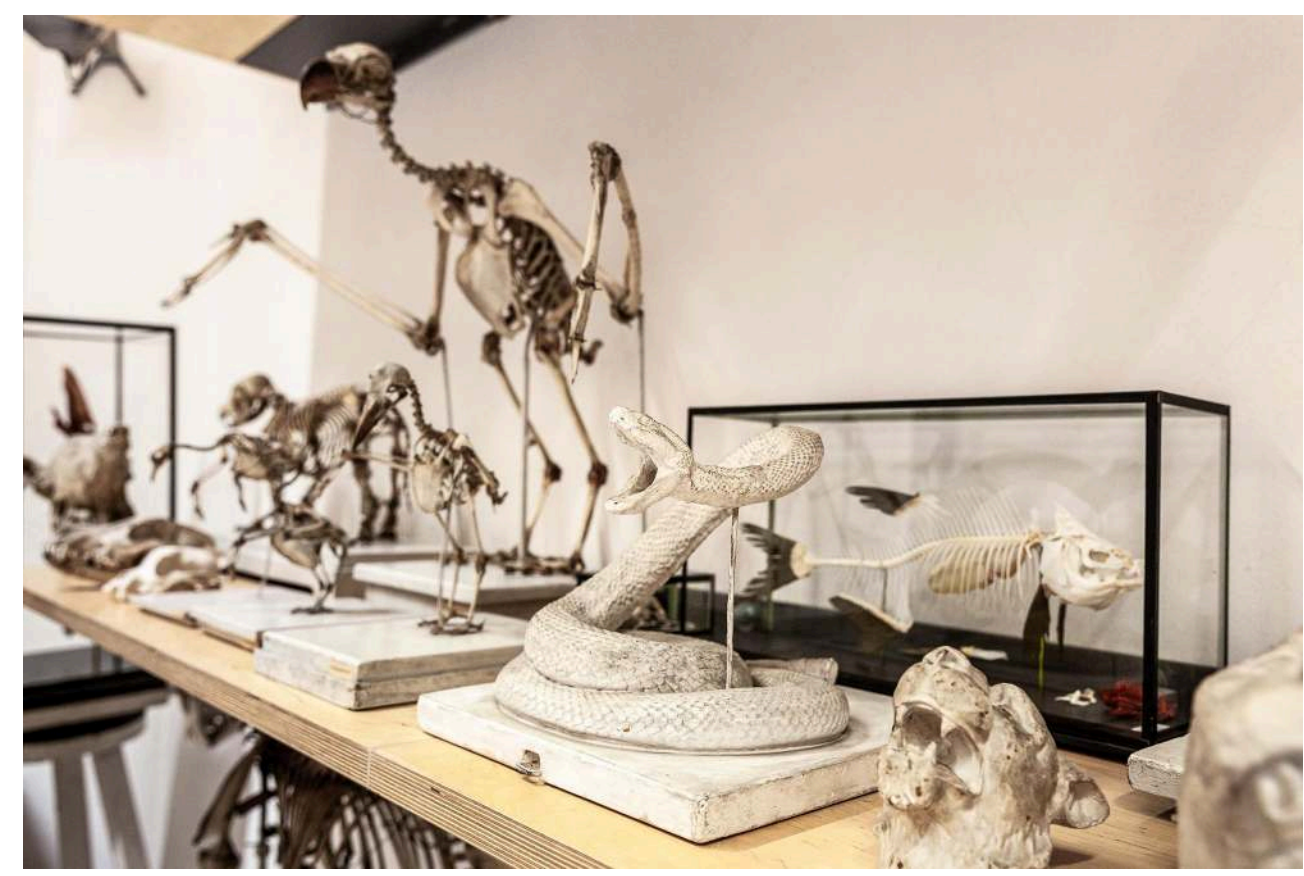

Modèles et spécimens d'anatomie animale : au premier plan, modèle en plâtre d'un serpent, réalisé par l'atelier de moulage artistique de Joseph Unger à Dresde.

(c) Robert Vanis.

Pour pouvoir mettre les objets «stables » de la collection à disposition des leçons de dessin anatomique, tout en protégeant tous les objets réputés «instables ", tous les spécimens et modèles ainsi évalués ont été marqués d'une plaquette de métal rouge (une forme d'interdiction interne). Comme mesure de protection supplémentaire, une vérification approfondie des socles anciens, dont les roulettes étaient en grande partie défectueuses ou grippées, a été effectuée. Les anciennes roulettes ont été démontées et les piédestaux d'origine ont été placés sur des piédestaux de substitution avec des roulettes à système de roulements à billes, faciles à utiliser [fig. 4]. Un dispositif de traction, conçu spécialement, et des plateaux tournants [fig. 5] pour les objets de petite et moyenne taille permettent un déplacement sans contact dans la pièce et sur les tables à dessin conçues à cet effet. 


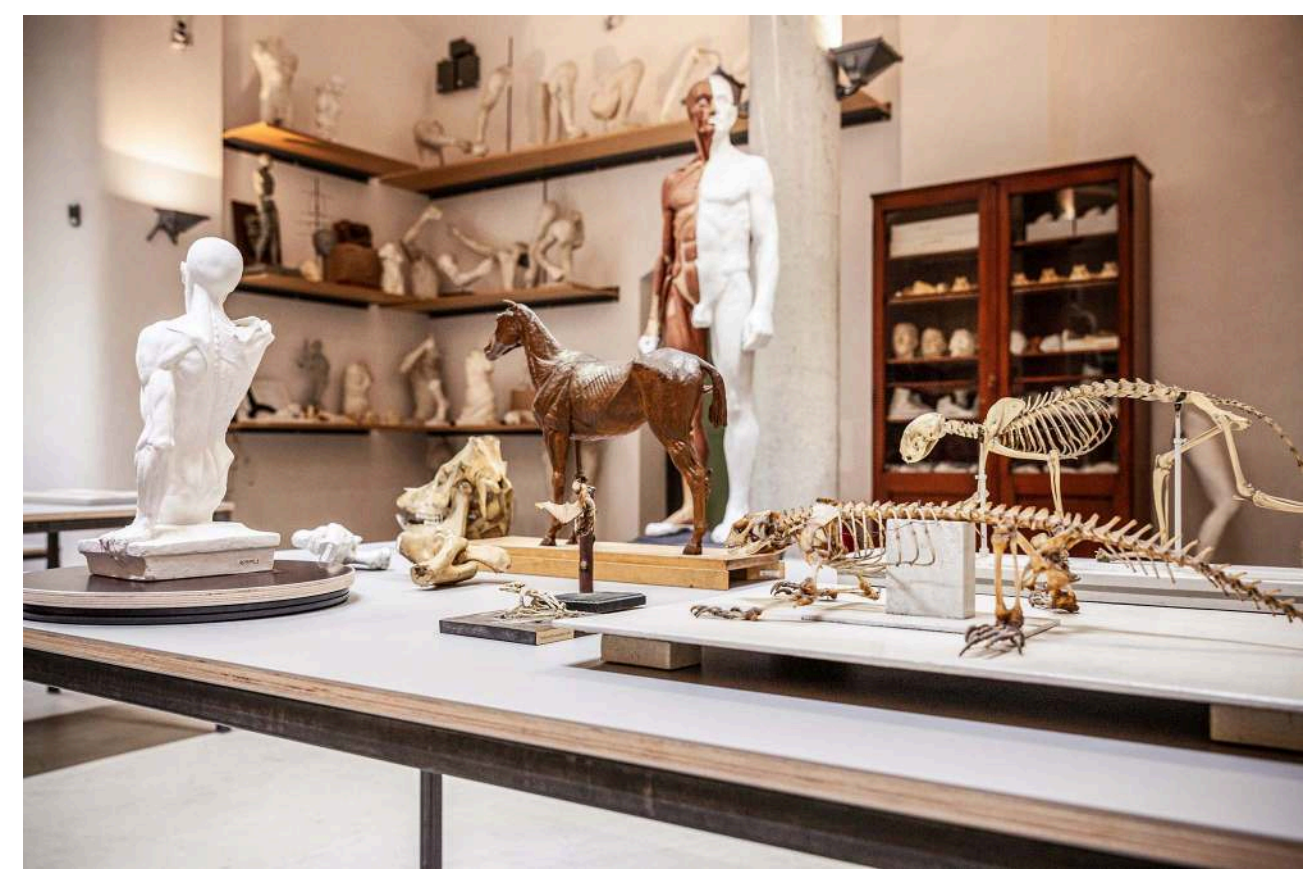

Modèles et spécimens d'anatomie animale et écorché anatomique humain en plâtre. Au centre, un modèle anatomique en réduction de cheval en plasticine, réalisé par le professeur Bammes entre 1975 et 1985 .

(C) Robert Vanis.

En 2018-2019, une restauration exemplaire de l'un des squelettes anciens à ligaments a été réalisée en coopération avec le programme d'étude "Technologie de l'art, conservation et restauration des objets d'art et du patrimoine culturel » de l'École supérieure. Comme tous les squelettes à ligaments de la collection, le Tireur d'épine présentait des traces d'usage et de vieillissement importantes, ainsi que des dommages causés par un resoclage. Outre les aspects liés à la conservation et aux matériaux, l'ensemble du processus de restauration a été envisagé sous l'angle d'une manipulation de ce spécimen qui soit correcte sur le plan éthique, la HfBK de Dresde ayant ainsi notablement contribué à la préservation d'autres spécimens humains anciens.

Les squelettes anciens à ligaments de la HfBK de Dresde ont été étudiés pour la première fois de 2011 à 2013 dans le cadre d'un mémoire de maîtrise, en coopération avec l'Académie royale danoise des beaux-arts de Copenhague (KADK). Au cours de ces travaux, de nombreux échantillons de matériaux ont été prélevés sur les squelettes à ligaments ${ }^{17}$ et des radiographies ont été réalisées ${ }^{18}$. Sur la base des résultats, il a été possible de localiser et d'identifier les matériaux introduits, tels que les huiles de séchage et les composés métalliques ajoutés a posteriori (mesures de restauration). En outre, les analyses en laboratoire ont montré que de la graisse s'échappait de l'intérieur de l'os, parfois massivement, et s'accumulait en surface. Ces résultats ont été publiés en $2018^{19}$.

$29 \mathrm{Au}$ cours des opérations de restauration actuelles, deux autres échantillons de matériaux ont été prélevés dans un premier temps, l'un sur une broche métallique (produit de corrosion) du genou du Tireur d'épine et l'autre, à titre de comparaison, sur un fil métallique (produit de corrosion) d'un os animal fortement graissé. L'analyse et l'évaluation des échantillons ont été menées par le laboratoire d'archéométrie de la 
HfBK de Dresde ${ }^{20}$. On a eu recours à la spectrométrie FT-IR5 (spectromètre infrarouge à transformation de Fourier, de type Bruker Tensor 27, et microscope de type IR Hyperion 2000, à cellule de diamant ou cristal ATR de germanium), à la microscopie électronique à balayage et à l'analyse par rayons $\mathrm{X}$ à dispersion d'énergie (MEB/EDX) (de type MEB Philips XL 30, avec détecteur de type Bruker EDX Xflash6I30). La composition des produits de corrosion indique que les acides gras de l'intérieur de l'os ont réagi avec les alliages métalliques, provoquant la formation de produits de corrosion vert-bleu (dont le carboxylate de cuivre). L'environnement acide formé à la surface et à l'intérieur de l'os, en lien avec le processus de vieillissement, détériore les composés calciques naturels des os et les produits de corrosion entraînent une décoloration irréversible de la substance osseuse. Pour ces raisons, les liaisons métalliques inappropriées utilisées ont été supprimées et remplacées par des assemblages en acier inoxydable et des joints collés.

Le nettoyage des os, des capsules et des ligaments a été effectué à l'aide d'un mélange d'acétone et d'éthanol dans un solvant, car le nettoyage à l'eau devait être exclu en raison de la forte dégradation du tissu organique. Le gonflement et le rétrécissement des fibres des tissus sous l'influence de l'eau auraient causé des dommages irréversibles et une nouvelle déstabilisation des connexions articulaires.

31 La manipulation des parties manquantes du corps (cinq dents et dix éléments des doigts et des pieds) a fait l'objet d'une discussion approfondie du point de vue éthique comme du point de vue de la restauration. Sur la base de considérations antérieures ${ }^{21}$, il a été décidé de reconstituer tous les os et dents perdus du Tireur d'épine et de présenter le modèle anatomiquement correct et correspondant à l'aspect originel. La reconstitution a été réalisée grâce à un mélange de cire, de résine et de craie; la peinture à l'huile a été utilisée pour la mise à la teinte.

Une autre opération de reconstitution concernait le socle du modèle. En raison d'une reprise ultérieure, le Tireur d'épine avait été monté dans une posture "assise" beaucoup trop basse, ce qui avait entraîné une forte déformation du corps. Sans enlever la structure de soutien ancienne, le socle a été légèrement surélevé et une substructure a été réalisée pour éviter de nouvelles détériorations des parties osseuses saillantes. 
Figure 6

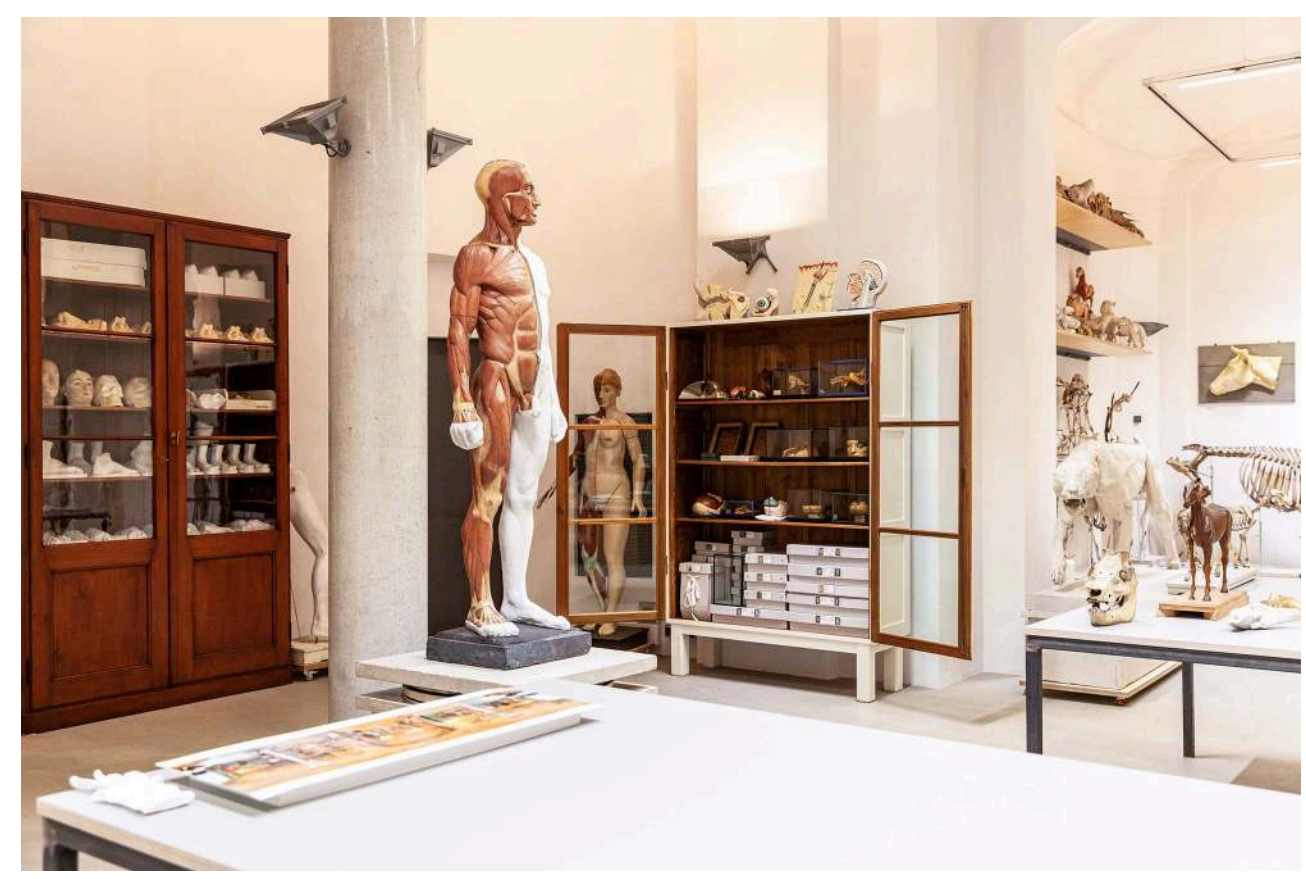

Salle de la collection anatomique : au centre, un modèle en plâtre coloré, grandeur nature, de musculature humaine, réalisé par Franz Andreas Naumann entre 1930 et 1935.

(C) Robert Vanis.

Toujours en 2018, une seconde restauration exemplaire a eu lieu dans le cadre du projet. Un relief de cheval en plâtre [fig. 6] grandeur nature, qui, selon l'état actuel des connaissances, a été réalisé en coopération avec l'Université royale de médecine vétérinaire de Saxe avant que celle-ci ne déménage à Leipzig en $1923^{22}$, présentait de graves dommages et des restaurations mal exécutées qui en ont considérablement altéré l'aspect extérieur. Les travaux de restauration ont été réalisés par des restaurateurs indépendants. Outre les opérations de nettoyage et de stabilisation, les restaurations antérieures ont dû être traitées. Plus précisément, il s'agissait de mastic de plâtre et de retouches très grossières, qui ont largement déformé l'anatomie du modèle. Grâce à une reprise ciblée des mastics et à des retouches précises, les parties visuellement gênantes ont pu être réintégrées dans l'aspect général du modèle.

Tous les travaux mentionnés se sont accompagnés d'une évaluation des concepts pédagogiques des cours d'anatomie actuels de la HfBK de Dresde, qui utiliseront de plus en plus la collection à l'avenir. En outre, des contenus d'enseignement et de recherche interdisciplinaires ont été formulés à partir de l'évaluation scientifique, en particulier dans les domaines de l'histoire de l'art et des collections ainsi que de la conservationrestauration.

\section{La collection de peintures}

La collection de peintures conservée par la HfBK de Dresde est à la fois un témoignage historique et une collection d'art. Au fil des siècles, elle s'est constituée à partir des activités d'enseignement de l'institution et comprend près de 1500 peintures. La plupart d'entre elles datent de l'époque de la République démocratique allemande (RDA), y compris de nombreux projets de recherche d'étudiants et des mémoires de 
diplôme, mais elles proviennent également de dons de professeurs à la retraite. On peut y trouver des œuvres plus anciennes, parmi lesquelles les tableaux dits de réception, avec lesquels les artistes ont candidaté à un poste d'enseignant à l'École supérieure, sont particulièrement remarquables. Ainsi, cette collection de peintures n'est pas seulement un témoignage du contenu de l'enseignement et des résultats artistiques de l'histoire de l'École supérieure des beaux-arts de Dresde : elle témoigne également de l'évolution des orientations formelles et du contenu de l'enseignement universitaire et de la pratique artistique en RDA, de la fondation du régime à sa chute.

Plus de 350 étudiants ont obtenu leur diplôme de peinture pendant cette période, parmi lesquels des artistes aussi connus que Karl-Heinz Adler, Eberhard Göschel, Angela Hampel, Siegfried Klotz, Gerda Lepke, Harald Metzkes, Stefan Plenkers, Cornelia Schleime, Strawalde, Christoph Wetzel et Walter Womacka. Ils ont été formés par des professeurs tels que Rudolf Bergander, Gerhard Bondzin, Jutta Damme, Fritz Eisel, Hans Grundig, Wilhelm Lachnit, Heinz Lohmar et Paul Michaelis, dont certains avaient euxmêmes été formés à la HfBK.

Pour une approche scientifique de la peinture de la RDA, la connaissance des artistes, de leur formation et de l'utilisation de tel ou tel matériau est indispensable dans des perspectives diverses. Étant donné que pour les nombreux étudiants qui ont été diplômés en peinture à Dresde entre 1950 et 1990, il subsiste au moins un support de leur travail de diplôme, qui fixe ainsi les résultats finaux de la formation de chaque artiste, la collection, jusqu'alors largement méconnue, s'impose presque comme un objet de recherche pour éclairer l'histoire de l'art de la $\mathrm{RDA}^{23}$.

$\mathrm{Au}$ début du projet, la conservation des œuvres était particulièrement précaire et la collection n'était accessible aux chercheurs et au public que de manière restreinte. Dans le cadre du projet, cette situation a pu être considérablement améliorée, entre autres dans le cadre d'une université d'été en $2017^{24}$, grâce à un inventaire professionnel et à un diagnostic d'ensemble, comprenant une documentation photographique (recto et verso avec échelle, pour chaque pièce) et des mesures de conservation-restauration. En outre, un plan général du dépôt des peintures a été dressé et un inventaire des locaux réalisé.

En adéquation avec la collection anatomique, pendant les six semaines de l'université d'été, chaque peinture a été analysée avec les six critères d'état déjà mentionnés et répertoriée dans la base de données des archives. Les 263 peintures de diplôme et les autres œuvres de sélection ont été examinées en détail : outre l'évaluation de l'état, une évaluation concrète des détériorations et une analyse des causes ont été effectuées. Dans certains cas, des recommandations ont aussi été formulées au cours de cette étape de travail en vue d'opérations de restauration ultérieures. En raison du grand nombre de peintures à examiner, il n'a pas été possible de dresser, pour chaque pièce, un protocole d'état individuel. En modifiant le masque de données, il a néanmoins été possible de stocker toutes les informations recueillies dans la base de données des $\operatorname{archives}^{25}$.

En plus ou parallèlement à cet état des lieux, des mesures de conservation préventive ont été prises. Dans un premier temps, les compartiments pour les peintures ont été nettoyés de la poussière et de la saleté et les fonds ont été recouverts de cartons de conservation. Les tableaux ont ensuite été triés selon leur taille et leur format et replacés dans les compartiments, les faces avant des tableaux étant chacune pourvue d'un intercalaire en papier de conservation, débordant, pour éviter de détériorer les 
couches picturales. Lors de la réinstallation des compartiments, on a veillé à ce que les numéros d'inventaire soient visibles sans déplacer les tableaux. Les peintures classées selon le critère "état instable, sauvegarde urgente » ont reçu un signalement spécial dans la base de données des archives. À l'avenir, ces œuvres seront consultables de préférence pour des études de conservation-restauration à la HfBK de Dresde et utilisées pour des formations et des travaux de diplôme et de séminaire ${ }^{26}$.

41 L'histoire de la collection et sa place dans l'histoire de l'art ont été revues de manière décisive et la collection a été érigée en sujet d'enseignement et de recherche dans les disciplines déjà mentionnées. Sur le thème des techniques picturales en usage en RDA, en particulier, les résultats du projet peuvent rayonner au-delà du territoire de Dresde. Il est ainsi possible de donner des informations, étayées par des analyses scientifiques et la technologie des radiations, sur les techniques de peinture utilisées en RDA qui, dans un contexte d'économie de rareté constante et de recherche d'alternatives, laissent espérer de nouvelles connaissances en histoire économique et culturelle ainsi qu'une contribution à la sauvegarde de l'art de la RDA.

\section{La présentation des résultats du projet}

42 Les résultats des travaux de recherche sur la collection de peintures de la HfBK Dresde ont été présentés en mai 2019 dans l'exposition «Avant l'art. La peinture à l'École supérieure des beaux-arts de Dresde de 1950 à 1990 ». Dans le prolongement de ce projet, l'Académie des beaux-arts de Saint-Pétersbourg a présenté des œuvres issues de la collection dans une exposition temporaire en novembre 2019.

Dans le cadre du colloque international « Collections des écoles supérieures des beauxarts : sauvegarder et transmettre ", la collection anatomique de la HfBK de Dresde a été rouverte officiellement le 6 novembre 2019. Depuis lors, des cours hebdomadaires de dessin anatomique ont été organisés pour les étudiants dans les salles rénovées et réaménagées à des fins de conservation. À l'avenir, la collection sera accessible au public intéressé quatre fois par semestre sur inscription préalable; des rendez-vous individuels et des visites guidées peuvent également être organisés pour les visiteurs et les groupes professionnels.

44 La cérémonie de réouverture au public de la collection anatomique s'est inscrite dans un programme de trois jours. Pour la première fois, les collections des écoles supérieures des beaux-arts en Europe ont été étudiées, en interrogeant leurs origines et leur statut dans les différentes écoles supérieures, les conditions de sauvegarde des résultats de l'enseignement artistique et de la recherche et leur impact ultérieur sur l'enseignement. L'objectif était de faire le point et de sonder le riche potentiel des collections pour l'enseignement, la recherche et le public, d'interroger les éventuelles tendances à la muséification et d'évoquer leur préservation et leur enrichissement.

Les actes du colloque, publiés au début de l'année 2020, sont accessibles sur le site internet ${ }^{27} \mathrm{du}$ projet. Ce site internet fournit documente le programme «Inventaire et évaluation de l'état des peintures et des mesures préalables de conservation dans les collections universitaires ", et accueille la publication Les restes humains en dépôt. Recommandations en matière de conservation et d'usage ${ }^{28}$. 


\section{NOTES}

1. Voir https://www.hfbk-dresden.de/hochschule/portraet/geschichte/ [lien valide en janvier 2021]. Voir également MÜHLENBEREND Sandra, Surrogate der Natur. Die historische Anatomiesammlung der Kunstakademie Dresden. Munich, Fink, 2007, p. 32.

2. PERES Constanze \& SCHMIDT Diether (dir.), Erneuerung als Tradition. 100 Jahre Dresdner Kunst und Kunstakademie im (inter)nationalen Zusammenhang, Dresde, Verlag der Kunst, 1997.

3. MÜHLENBEREND Sandra, Surrogate der Natur.

4. MÜHLENBEREND Sandra, Surrogate der Natur, p. 32.

5. MÜHLENBEREND Sandra, Surrogate der Natur, p. 44.

6. Voir MÜHLENBEREND Sandra, «Präparate unter Verdacht. Künstleranatomie zwischen 1933-1945 an der Hochschule für Bildende Künste Dresden », in MÜHLENBEREND Sandra, FUCHS Jakob \& MARUŠIĆ Vera (dir.), Unmittelbarer Umgang mit menschlichen Überresten in Museen und Universitätssammlungen. Statements und Fallbeispiele, Dresde, HfBK, 2018, p. 55-63, ici p. 55 [disponible en ligne] https://wissenschaftliche-sammlungen.de/files/1815/4469/5645/ Unmittelbarer-Umgang-mit-menschlichen-berresten-in-Museen-und-

Universittssammlungen.pdf [lien valide en janvier 2021].

7. Les squelettes à ligaments étaient à l'origine conçus en grande partie sans recourir à des apports artificiels tels que des fils métalliques notamment. Les connexions articulaires naturelles (capsules et ligaments) ont été laissées sur l'os à cette fin.

8. MÜHLENBEREND Sandra, Surrogate der Natur, p. 178.

9. MÜHLENBEREND Sandra, Surrogate der Natur, p. 154.

10. MÜHLENBEREND Sandra, Surrogate der Natur, p. 80.

11. MÜHLENBEREND Sandra, Surrogate der Natur, p. 222.

12. MÜHLENBEREND Sandra, Surrogate der Natur, p. 100.

13. MÜHLENBEREND Sandra, Surrogate der Natur.

14. Les radiographies ont été réalisées par le professeur Ivo Mohrmann et par Kerstin Riße (HfBK de Dresde).

15. L'analyse et l'évaluation des matériaux ont été réalisées par le professeur Christoph Herm et par Annegret Fuhrmann (HfBK de Dresde).

16. La documentation photographique a été réalisée par Maria Katharina Franz (HfBK de Dresde).

17. Les mesures et l'évaluation ont été réalisées par le professeur Christoph Herm, Annegret Fuhrmann et Sylvia Hoblyn (HfBK de Dresde).

18. Les radiographies ont été réalisées par le professeur Ivo Mohrmann, Kerstin Riße et Monika Kammer (HfBK de Dresde).

19. FRANK Steen K., MEYER Ion, HERM Christoph \& BOTFELDT Knud B., «Status report, conservation and exhibition proposal for 14 natural skeletons from the anatomical collection at the University of Fine Arts in Dresden ", VDR Beiträge zur Erhaltung von Kunst- und Kulturgut, $\mathrm{n}^{\circ} 1$, 2018, p. 71-86.

20. Les mesures et l'évaluation ont été réalisées par Janine Kaden (HfBK de Dresde).

21. FUCHS Jakob, «Umgang mit fehlenden Körperteilen an historischen Skelettaufstellungen », in MÜHLENBEREND Sandra, FUCHS Jakob \& MARUŠIĆ Vera (dir.), Unmittelbarer Umgang mit menschlichen Überresten..., p. 192-200.

22. MÜHLENBEREND Sandra, Surrogate der Natur, p. 243.

23. Voir https://artonomia.de/wp-content/uploads/2019/05/Liste-derAbsolvent_innen-1947-1990_final.pdf [lien valide en janvier 2021].

24. La gestion et l'encadrement ont été assurés par le professeur Ivo Mohrmann et par Kathleen Rosenthal (HfBK de Dresde). 
25. Dans le cadre du projet, les données, les résultats des recherches et les photographies de la collection anatomique et de la collection de peintures ont ainsi été entièrement compilés dans la base de données des archives Augias.

26. Voir aussi: ARBEITSGRUPPE BMBF-PROJEKT „KÖRPER UND MALEREI“, Handreichung. Bestands- und Zustandserfassung von Gemälden sowie konservatorische Erstmaßnahmen in universitären Sammlungen, Dresde, HfBK, 2018 [disponible en ligne], https://wissenschaftliche-sammlungen.de/ download_file/view/1645/ [lien valide en janvier 2021].

27. https://artonomia.de/wp-content/uploads/2020/06/Sammlungen_an_Kunsthochschulen.pdf [lien valide en janvier 2021].

28. FUCHS Jakob, GABLER Diana, HERM Christoph, MARKERT Michael et MÜHLENBEREND Sandra, Menschliche Überreste im Depot. Empfehlungen für Betreuung und Nutzung [en ligne], Dresde, HfBK, 2020, https://wissenschaftliche-sammlungen.de/files/3515/7987/3438/ Menschliche_berreste_im_Depot.pdf [lien valide en janvier 2021].

\section{RÉSUMÉS}

L'École supérieure des beaux-arts de Dresde (HfBK) est, avec l'École des beaux-arts de Paris, l'une des deux académies des beaux-arts en Europe qui possède une collection ancienne et très riche pour l'anatomie artistique. La collection d'anatomie de l'École supérieure des beaux-arts de Dresde a été constituée depuis le début du XIX ${ }^{\mathrm{e}}$ siècle et constamment enrichie. Elle comprend aujourd'hui près de 700 modèles anatomiques et spécimens d'anatomie humaine et animale. Grâce à ces nombreux spécimens et modèles anciens, les professeurs ont développé au $\mathrm{xx}^{\mathrm{e}}$ siècle pour l'enseignement du dessin anatomique leurs propres supports et méthodes pédagogiques, dont la valeur a été reconnue largement au-delà des frontières de la HfBK Dresden. Après 1989, les modèles et spécimens anciens ont beaucoup perdu de leur importance ; la collection n'a plus guère été utilisée pour l'enseignement et son état de conservation s'est détérioré. Ce n'est qu'à la fin des années 1990 que l'on a redécouvert la valeur immense de la collection et que l'on a pris les premières mesures de protection et mené les premières études. La collection a d'abord été complètement retirée de l'enseignement actif et elle a été placée dans une salle de dépôt. Depuis lors, ses fonds ont fait l'objet de recherches en histoire de l'art et en conservation. De février 2017 à janvier 2020, un projet financé par le ministère fédéral allemand de l'Éducation et de la Recherche a eu pour objectif de rendre la collection à nouveau disponible pour l'enseignement, après toutes ces années. Le 6 novembre 2019, après trois ans de recherches intensives et de projets, on a fêté sa réouverture.

Dresden University of Fine Arts (HfBK) is, with the École nationale des beaux-arts de Paris, one of the two academies of Fine Arts in Europe that possesses an old and very rich collection for artistic anatomy. The anatomy collection of Dresden University of Fine Arts has been built up since the beginning of the 20th century and constantly expanded. It comprises today nearly 700 anatomic models and specimens of human and animal anatomy. Thanks to those numerous specimens and ancient models, teachers developed in the 20th century their own educational material and methods whose value had been recognized far beyond the frontiers of the HfBK Dresden. After 1989, the old models and specimens lost their importance; the collection was hardly used for teaching and its condition had deteriorated. It was only in the late 1990's that the immense value of the collection was rediscovered, and that the first protective measures were 
taken and the first studies were carried out. The collection was first removed completely from active teaching material and placed in a storage room. Since then, those fonds have been the focus of research in art history and conservation. From February 2017 to January 2020, a project financed by the Federal Ministry of Education and Research aimed at making the collection available again for teaching, after all those years. On 6 November 2019, after three years of intensives research and projects, its reopening was celebrated.

Die Hochschule für Bildende Künste in Dresden (HfBK) ist neben der École nationale des beauxarts in Paris eine von zwei Kunstakademien in Europa, die über eine historische und sehr umfangreiche Sammlung zur Künstleranatomie verfügt. Die Anatomische Sammlung der HfBK Dresden wurde seit Beginn des 19. Jahrhunderts kontinuierlich zusammengetragen und erweitert. Sie umfasst heute ca. 700 anatomische Modelle und Präparate zur Human- und Tieranatomie. Mit Hilfe der zahlreichen historischen Präparate und Modelle entwickelten die Professoren für den anatomischen Zeichenunterricht im 20. Jahrhundert eigenen didaktische Modelle und Lehrmethoden, die in ihrer Bedeutung weit über die Grenzen der HfBK Dresden hinausgingen. Nach 1989 verloren die historischen Modelle und Präparate stark an Bedeutung; die Sammlung wurde kaum mehr für den Unterricht genutzt und ihr Erhaltungszustand verschlechterte sich. Erst Ende der 1990er Jahre wurde der enorme Wert der Sammlung wiedererkannt und erste Maßnahmen zu ihrem Schutz und ihrer Erforschung eingeleitet. Zunächst wurde die Sammlung vollständig aus dem aktiven Lehrbetrieb herausgenommen und in einen Depotraum verbracht. Seither steht ihr Bestand im Fokus der kunsthistorischen und konservatorischen Erforschung. Von Februar 2017 bis Januar 2020 fand ein vom Bundesministerium für Bildung und Forschung gefördertes Projekt statt, mit dem Ziel, die Anatomische Sammlung nach vielen Jahren wieder für die Lehre zur Verfügung zu stellen. Am 06.11.2019 fand nach dreijähriger intensiver Forschungs- und Konzeptarbeit die feierliche Wiedereröffnung statt.

\section{INDEX}

Keywords : University of Fine Arts of Dresden, Körper und Malerei, anatomic models, anatomy collection, drawing, teaching, Dresden, painting, painting collection

Schlüsselwörter : Hochschule für Bildende Künste Dresden, Körper und Malerei, anatomische Modelle, Anatomische Sammlung, Zeichnung, Lehre, Dresden, Malerei, Gemäldesammlung

Mots-clés : École des beaux-arts de Dresde, Körper und Malerei, modèles anatomiques, préparations anatomiques, dessin, enseignement, Dresde, peinture, collection de peinture

\section{AUTEURS}

\section{SANDRA MÜHLENBEREND}

Chef de projet et coordinatrice « Körper und Malerei » (février 2017-janvier 2020), École des

beaux-arts de Dresde

sandra.muehlenberend@web.de

\section{JAKOB FUCHS}

Assistant scientifique, projet « Körper und Malerei » (février 2017-janvier 2020), École des beauxarts de Dresde

fuchs@hfbk-dresden.de 


\section{IVO MOHRMANN}

Président du comité de pilotage de projet « Körper und Malerei » (février 2017-janvier 2020), chef du département de Technologie de l'art, d'Examen radiologique et de Photographie, conservateur des collections universitaires, École des Beaux-Arts de Dresde

mohrmann@hfbk-dresden.de 